\title{
34. REMARKS ON LOCAL STRUCTURE AND KINEMATICS
}

\author{
A. BLAAUW
}

Kapteyn Laboratory, Groningen, The Netherlands

\begin{abstract}
Attention is drawn to a few aspects of the state of motion of the local population which may become of importance for the study of local spiral structure. Uncertainties in the present knowledge of the local standard of rest are discussed, (a) with regard to the possible outward or inward motion with respect to the galactic centre, and (b) with regard to the component in the direction of circular motion. We furthermore draw attention to the quiet state of motion of the local interstellar gas and to the contrasting fairly high group velocities among the recently formed $\mathrm{OB}$ associations.
\end{abstract}

\section{Introduction}

Studies of the velocity distribution and space distribution of youngest stars and gas must now be regarded in the context of the very useful hypothesis of the density wave theory for spiral structure, which must be expected to lead to certain predictions with regard to the state of motion of these objects in and outside the spiral structure. For the understanding of the properties of the local population this theory should be expected to refer mostly to the Orion arm. The significance of the Orion arm in the context of the density wave theory is not quite clear, the indications are that it must be considered as a secondary feature. For such studies the following remarks may become useful.

\section{The Local Deviation from Circular Motion}

The radioastronomical as well as the optical radial velocity observations are always referred to the sun, and, normally, subsequently reduced to the 'local standard of rest' (LSR), i.e. the circular velocity around the galactic centre. This reduction is done by means of an assumed value of the velocity, $S$, of the sun with respect to the LSR. The velocity $S$ is not accurately known and its uncertainty in amount and direction are involved in such problems as: the possible common motion of gas and stars in the radial direction from the galactic centre outward or inward (general expansion or contraction), and the deviation from circular velocity for the velocity component in the direction of circular motion for these objects but especially for the gas (the possible lag of the gas). The following reviews the present uncertainties in these quantities.

Figure 1 represents the velocities projected on the galactic plane with respect to the sun: $U$ is the component toward $l^{\mathrm{II}}=180^{\circ}$ (anticentre); $V$, toward $l^{\mathrm{II}}=90^{\circ}$ (galactic rotation). The various points indicate the generally adopted values of $U$ and $V$ for the 'common' types of stars, giants and dwarfs of spectral types $G, K$ and $M$ taken from the compilation by Delhaye (1965), for the interstellar medium as a mean of various determinations (Takakubo, 1967; Venugopal and Shuter, 1967; Blaauw, 1952), and for the OB stars within $600 \mathrm{pc}$ from Lesh (1968). The accuracy of these positions 
is 1 to $2 \mathrm{~km} \mathrm{~s}^{-1}$. For the spectral types $A$ and $F$, the velocity pattern is rather patchy and can best be described as a superposition of a number of discrete streamings. The hatched sections of the diagram represent the approximate areas of these groupings for the A-type stars according to Eggen (1963).

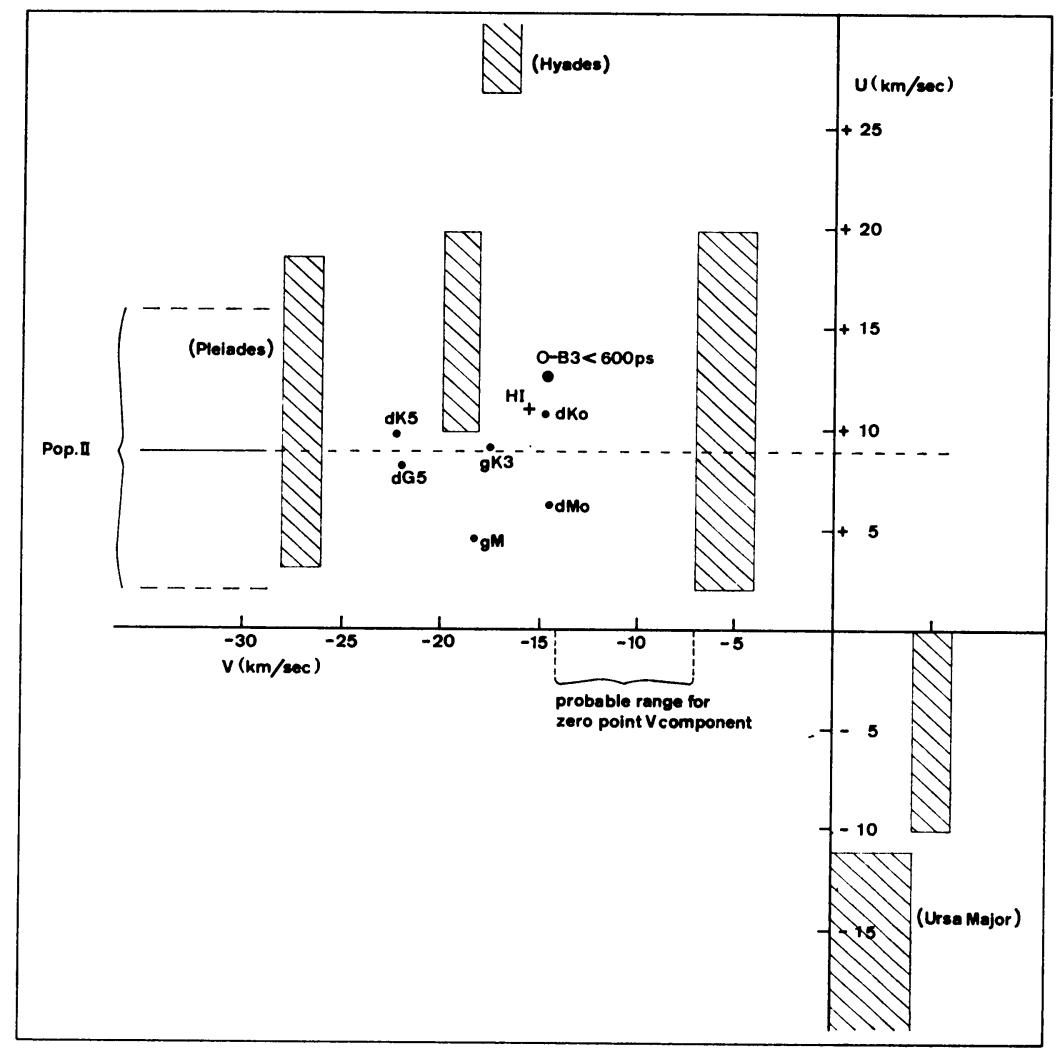

Fig. 1. Velocity, with respect to solar motion, for the late-type 'common stars' and for the neutral hydrogen and the O-B3 stars within $600 \mathrm{pc}$. Hatched areas indicate principal streamings in A-type stars. Only the components $U$ (toward galactic anticentre) and $V$ (direction of galactic rotation) are shown.

\section{A. VELOCITY WITH RESPECT TO GALACTIC CENTRE}

Since no stellar, nor gaseous objects located at the galactic centre can be reliably used (only radio measures are available and they concern objects in the interstellar medium affected by strong turbulent motion), the most direct method consists of using those stellar objects which may be expected to show no systematic expansion or contraction. This is the case for objects belonging to Population II and to the old Disk Population. We list below 4 categories for which a solution of the velocity component $U$ can be derived from the literature. 
RR Lyrae Variables, periods $>0.42 d$ (Van Herk, 1965):

$$
U=+23 \mathrm{~km} \mathrm{~s}^{-1} \pm 9 \text { (p.e.) from } 76 \text { stars; }
$$

Subdwarfs (Deeming, 1961):

$$
U=+2 \mathrm{~km} \mathrm{~s}^{-1} \pm 18 \text { (p.e.) from } 103 \text { stars; }
$$

Planetary Nebulae (Minkowski, 1965):

$$
U=+4 \mathrm{~km} \mathrm{~s}^{-1} \pm 6 \text { (p.e.) from } 136 \text { objects; }
$$

Globular Clusters (Kinman, 1959):

$$
U=+10 \mathrm{~km} \mathrm{~s}^{-1} \pm 18 \text { (p.e.) from } 70 \text { clusters. }
$$

The differences between these determinations cannot be regarded as significant. A weighted mean value is:

$$
\langle U\rangle=+9 \mathrm{~km} \mathrm{~s}^{-1} \pm 5 \text { (p.e.). }
$$

This mean value, also represented in Figure 1, lies within the range of those for the various groups in that figure. There is, therefore, no evidence of expansion or contraction of these objects. However, the uncertainty is large and does not preclude a possible expansion or contraction of $10 \mathrm{~km} \mathrm{~s}^{-1}$ or less. An expansion of $+7 \mathrm{~km} \mathrm{~s}^{-1}$ for matter in the solar neighbourhood, as proposed by Kerr (1962) in order to bring $21 \mathrm{~cm}$ observations in the first and fourth galactic quadrants on a common rotation curve, therefore cannot be ruled out or confirmed.

Obviously in view of the importance of this problem, attempts should be made to improve upon the determination of $\langle U\rangle$. At first thought, the high velocity objects in the disk population might seem to be an additional group for which the mean value of $U$ should be added to those listed above, since their velocity pattern might be expected to be smoothed out and symmetric in the $U$ component. This should be the case especially if the choice is limited to strong negative values of $V$, for instance $V<-50 \mathrm{~km} \mathrm{~s}^{-1}$ because then we are dealing with the more eccentric orbits. It appears that such a choice is not a suitable one because the distribution of the $U$ components is definitely asymmetric with an excess of positive values of the component $U$. This is shown, for instance, in the distribution of projected space velocities of the long period variables with periods shorter than 300 days, see Osvalds and Risley (1961), and also apparent if one plots the distribution of the $U$ components for the $K$ giants with $V<-50 \mathrm{~km} \mathrm{~s}^{-1}$ in the catalogue of Eggen (1966). Another demonstration of this asymmetry may be found in Miczaika's plot of the distribution of the apices of the high velocity stars as a function of galactic longitude of which a reproduction may be found in Oort's paper on stellar dynamics (Oort, 1965). This asymmetry is not yet well understood. It may well be related to the non-axisymmetrical gravitational field in the galactic plane. Further efforts to improve upon the determination of $\langle U\rangle$ therefore should be based preferably on old population stars with inclined orbits with respect to the galactic plane. 


\section{B. POSSIBLE LAG WITH RESPECT TO CIRCULAR VELOCITY}

Figure 1 shows that the $V$ components for the common stars are about $-18 \mathrm{~km} \mathrm{~s}^{-1}$. Since no direct way exists to measure the sun's velocity component $V$ with respect to the local standard of rest, an indirect estimate must be made. Axisymmetric dynamical theory for a well mixed steady state stellar system predicts a systematic lag with respect to the circular velocity for a given component of the stellar population as a consequence of the dispersion of the stellar velocities with respect to circular motion, and of the density gradient with increasing distance from the galactic centre. Denoting the logarithmic density gradient by $\delta \log v / \delta R$, the lag by $T$, the circular velocity by $\theta_{\mathrm{c}}$, and the dispersion of stellar velocity components in the direction of $U$ by $\tau_{U}$, we have

$$
\frac{\delta \log v}{\delta R}=T \frac{2 \theta_{\mathrm{c}}-T}{\tau_{U}^{2}}+\text { small known terms . }
$$

The estimate of the density gradient is quite uncertain; on the one hand we know that the older population among the common stars probably has a gradient $\delta \log v / \delta R$ between -0.15 and -0.30 from studies at intermediate latitudes. But on the other hand the younger population may well show a density distribution related to the local spiral structure. If we use the value for the old component, we arrive at values of $T$ such that, if added to the $V$ components in the figure, they place the circular velocity point in the range between $V=-7$ and $-14 \mathrm{~km} \mathrm{~s}^{-1}$. This is indicated in the figure. If we are dealing with a different density gradient for the younger star population, the possible range may well shift several $\mathrm{km}$ farther towards more negative values of $V$.

It appears from the figure that a systematic lag for the neutral hydrogen in the solar neighbourhood and for the closely associated youngest stars (O-B 3) of up to $8 \mathrm{~km} \mathrm{~s}^{-1}$ may well exist but it may also be negligible. The situation may be improved by systematic observations of the radial velocities of older population stars at intermediate galactic latitudes at longitudes $90^{\circ}$ and $270^{\circ}$.

\section{Local State of Motion of Gas and Youngest Stars}

I should like to stress two points: the remarkably quiet state of motion of the local gas and the, in comparison to this, much more turbulent state of motion in the youngest stellar population.

\section{A. STATE OF MOTION OF LOCAL GAS}

The most complete information on the motion of the local hydrogen comes from the $21 \mathrm{~cm}$ observations. At the lowest latitudes there is some difficulty in distinguishing nearer and more distant gas. It is therefore most instructive to consider the information derived from intermediate latitudes; reference is made in particular to the analysis of $21 \mathrm{~cm}$ profiles by Takakubo (1967). His Figures 1-7, dealing with the latitudes $+25^{\circ}$, $+20^{\circ},+15^{\circ},+10^{\circ},-10^{\circ},-15^{\circ}$ and $-20^{\circ}$ clearly reveal the sinusoidal variation of 
the radial velocity of the main gaussian component notwithstanding the fact that the semi-amplitude on the average is only $3.8 \mathrm{~km} \mathrm{~s}^{-1}$. There are no pronounced disturbances in the low velocity field. Fairly high negative velocities are observed at the galactic longitudes about $90^{\circ}$ to $160^{\circ}$ which partly must be ascribed to matter at large distances from the plane above the Perseus arm. Takakubo's figures may be considered to be representative for the volume up to about $400 \mathrm{pc}$ from the sun. This includes most of the system of the Gould Belt of early type stars. Its existence also in the distribution of neutral hydrogen is well established, particularly by the excess densities at positive latitudes towards the galactic centre and negative latitudes toward the anticentre. In these directions outward motions in the neutral hydrogen, probably associated with the Gould Belt system, are observed, but the main feature is that of the differential galactic rotation.

\section{B. STATE OF MOTION OF YOUNGEST STARS}

The youngest stars, spectral types O to B 3 are known to have smaller velocity dispersion than the later, older types. A.r.m.s. velocity in one component of about $10 \mathrm{~km} \mathrm{sec}^{-1}$ is usually found for the stars within, say, $600 \mathrm{pc}$. It would be wrong, however, to conclude from this that no pronounced streamings occur. This stellar population is known to be built up of a number of more or less isolated associations plus the somewhat older field stars. The nearest associations, Scorpio Centaurus, Per OB II, Ori OB I, Lac OB I, Cep OB II, Cep OB III, etc. contain most of the stars of types $\mathrm{O}$ to $\mathrm{B} 2$ and about half of those of types B 3. Whereas within each of these associations the internal velocity dispersion is very small, a few $\mathrm{km} \mathrm{s}^{-1}$ at most, the associations as a whole have considerable relative velocities. Thus, the association Per OB II has a relative velocity, with respect to the average B star population, of about $15 \mathrm{~km} \mathrm{~s}^{-1}$, and the average velocity of Scorpio Centaurus is about $8 \mathrm{~km} \mathrm{~s}^{-1}$, and these two motions are approximately opposite. The velocity of the Orion association also is about $10 \mathrm{~km} \mathrm{~s}^{-1}$. The differential galactic rotation, so clearly visible in the interstellar gas, is only a secondary phenomenon among the $\mathrm{O}$ to $\mathrm{B} 3$ stars within $600 \mathrm{pc}$ and the apparently irregular velocity pattern of the principal groupings prevails. One strongly gets the impression that in the process of star formation out of a quiet interstellar medium fairly large group motions are conveyed to the resulting associations. Conversely the state of motion of the youngest stars with this fairly high irregularity, is not necessarily an indication of large turbulence in the generating interstellar medium. For descriptions of the state of motion of the nearest O to B 3 stars, see Blaauw (1956), and Lesh (1968).

It may be noted that these large group velocities of the associations which imply considerable deviation from circular velocity, would, as seen from a distance, lead to quite erroneous location in the Galaxy if the standard relation between distance and radial velocity based on circular velocity is used. The Per OB II association, as seen from the Perseus arm would be misplaced by almost a kiloparsec. This implies that also distance determinations of distant associations by means of the standard galactic rotation curve may involve considerable accidental errors. 


\section{References}

Blaauw, A.: 1952, Bull. Astron. Inst. Netherl. 11, 459.

Blaauw, A.: 1956, Astrophys. J. 123, 408.

Deeming, T. J.: 1961, Monthly Notices Roy. Astron. Soc. 123, 273.

Delhaye, J.: 1965, Stars and Stellar Systems 5, 61.

Eggen, O. J.: 1963, Astron. J. 68, 697.

Eggen, O. J.: 1966, Roy. Obs. Bull., No. 125.

Kerr, F. J.: 1962, Monthly Notices Roy. Astron. Soc. 123, 327.

Kinman, T. D.: 1959, Monthly Notices Roy. Astron. Soc. 119, 559.

Lesh, J.: 1968, Astrophys. J. Suppl. Ser. 17, 371.

Minkowski, R.: 1965, Stars and Stellar Systems 5, 321.

Oort, J. H.: 1965, Stars and Stellar Systems 5, 455.

Osvalds, V. and Risley, A. M.: 1961, Publ. Leander McCormick Obs. 11, 147.

Takakubo, K.: 1967, Bull. Astron. Inst. Netherl. 19, 125.

Van Herk, G.: 1965, Bull. Astron. Inst. Netherl. 18, 71.

Venugopal, V. R. and Shuter, W. L. H.: 1967, Astron. J. 72, 534. 